\title{
Identification and Validation of Aurora Kinase A (AURKA) Involved in the Progression and Imatinib Resistance of Advanced Gastrointestinal Stromal Tumors
}

\section{Xiaobin Cheng ( $\sim 1512016 @ z j u . e d u . c n)$}

Zhejiang University

Jinhai Wang

Zhejiang University School of Medicine First Affiliated Hospital

\section{Sen Lu}

Zhejiang University School of Medicine First Affiliated Hospital

\section{Weina Fan}

Zhejiang University School of Medicine First Affiliated Hospital

\section{Weilin Wang}

Zhejiang University School of Medicine Second Affiliated Hospital

\section{Research}

Keywords: AURKA, Progression, Imatinib resistance, Gastrointestinal stromal tumors

Posted Date: September 23rd, 2020

DOI: https://doi.org/10.21203/rs.3.rs-79473/v1

License: (c) (1) This work is licensed under a Creative Commons Attribution 4.0 International License.

Read Full License 


\section{Abstract}

Background: Gastrointestinal stromal tumor (GIST) is a common tumor originating from mesenchyme in the alimentary system. GISTs exhibit unique malignant behavior compared to typical carcinomas. Here, we explored genes that drive the progression and imatinib resistance of GISTs by bioinformatic tools and experiments.

Methods: We analyzed gene expression profiles of GISTs and identified a key gene, AURKA, which regulated the progression of GISTs. We further determined the clinical significance of AURKA by immunohistochemical $(\mathrm{IHC})$ staining assays and survival analysis. A series of in vitro experiments were performed to investigate in detail the effect of AURKA on the aggressiveness and imatinib resistance of GIST cells.

Results: Our results showed that AURKA was higher in advanced GIST tissues than in non-advanced GIST tissues. AURKA mainly involved in cell cycle related biological processes as revealed by GSEA. AURKA was positively related with GIST progression and could be an independent prognostic marker of GISTs. It was also found to exhibit consistent expression patterns in several human malignancies. Additionally, AURKA influenced gene mutations associated with imatinib resistance. Overexpression of AURKA in GIST cells promoted cell proliferation and apoptosis, even after administration of imatinib. But imatinib significantly inhibited the effects of AURKA overexpression on the migration of GIST cells.

Conclusions: Bioinformatics analyses and subsequent experiments identified $A U R K A$ as a key gene in GIST progression. AURKA showed a contribution to imatinib resistance and could be a candidate therapeutic target for GISTs.

\section{Background}

Gastrointestinal stromal tumor (GIST) is the most common mesenchymal tumor of the alimentary system and originates from the interstitial cells of Cajal (ICC) ${ }^{[1]}$. About $71 \%$ of GISTs present with KIT mutations (71\%) or PDGFRa (14\%) mutations, leaving approximately $10 \%-15 \%$ of GISTs without any KIT or PDGFRa mutations and the remaining are reported as KIT/ PDGFRa wild type $\mathrm{e}^{[2,3]}$. The malignant potential of GIST is stratified based on its tumor size, mitotic index, and location according to the modified NIH criteria. Based on these criteria, GISTs are classified as high-risk, intermediate-risk, low-risk, and very low-risk ${ }^{[4]}$. The 5 -year survival rate of patients with malignant GISTs ranges from $35-65 \%[5]$. Surgical resection is the mainstay treatment for primary localized GISTs. However, even though a histopathologically complete GIST tumor resection is done, the recurrence rate is $40 \%-80 \%[5]$. The median time to recurrence for most patients is approximately $12-16$ months $^{[6]}$. The administration of imatinib, a tyrosine kinase inhibitor (TKI) that targets KIT and PDGFRa, has improved the prognosis in GIST patients. Studies have reported that imatinib can achieve disease control in about $70 \%-85 \%$ of patients with advanced KIT-positive GISTs ${ }^{[1]}$. However, imatinib has limited efficacy in eradicating mature GIST cells and studies have suggested GIST persistence in prolonged TKI therapy ${ }^{[1]}$. About $85 \%-90 \%$ of 
patients usually experience disease progression within 20-24 months due to acquired resistance to imatinib $^{[2,3,7]}$. For imatinib-resistant GISTs, other TKIs or novel molecular targeted therapies such as sunitinib, regorafenib, HSP90 inhibitors, and IGFIR inhibitor have been developed and approved ${ }^{[8-11]}$. Therefore, novel therapeutic targets are beneficial for advanced and imatinib-resistant GISTs.

Currently, bioinformatics methods are being used to explore molecular signatures associated with progression and clinical outcomes in several types of malignancies ${ }^{[12,13]}$. In this study, the gene sets GSE136755 and GSE47911 from the Gene Expression Omnibus (GEO) were used to explore key molecules that highly contribute to GIST progression. To eliminate selection bias, the gene expression profiles of GISTs with KIT mutations were extracted and analyzed. AURKA was identified as a key gene among several hub genes in the GSE136755 dataset and further validated in the GSE47911 dataset. Immunohistochemistry, survival analysis, and in vitro experiments were performed to confirm the association between AURKA expression and malignant signatures of GISTs.

\section{Methods}

\section{Gene sets acquisition and identification of DEGs}

GSE136755 gene set was obtained from the Gene Expression Omnibus (GEO) (https://www.ncbi.nlm.nih.gov/geo/). The GSE136755 was based on the GPL17077 platform (Agilent039494 SurePrint G3 Human GE v2 8x60K Microarray 039381) and included clinicopathological information of 65 Homo Sapiens GIST tumor specimens without preoperative treatment, such as imatinib. The primary GISTs with KIT mutations were selected to further analysis of differentially expressed genes (DEGs). High-risk GISTs were defined as advanced samples, which included 16 samples, while thirty-one low-risk and very low-risk GISTs were considered as non-advanced samples. The intermediate-risk GISTs were not included since they were not specified in terms of their biological behavior compared to the high-risk and low-risk to GISTs. GEO2R, an R-associated web tool supplied by the National Center for Biotechnology Information, was applied to filter the DEGs between advanced and non-advanced GISTs. DEGs were identified using the cutoff: |log2FoldChange| $>1$ and adju. P-value < 0.05. The DEGs were reserved in text format for hierarchical clustering analysis in Morpheus (https://software.broadinstitute.org/morpheus).

\section{Functional enrichment analysis of DEGs}

The Database for Annotation, Visualization, and Integrated Discovery (DAVID) (http://david.ncifcrf.gov, version 6.8) is a web bioinformatics resource used to extract functional annotation information of genes. Gene ontology (GO) is a major bioinformatics tool for gene annotation and analysis. Kyoto Encyclopedia of Gene and Genome (KEGG) is a popular database for analysis of advanced gene functions and potential signaling pathways in large-scale molecular data. DAVID was used to perform GO and KEGG enrichment analyses of DEGs. The cut-off criterion was a false discovery rate (FDR) $<0.05$.

\section{Protein-protein interaction (PPI) network and module analysis}


The Search Tool for the Retrieval Interacting Genes (STRING) (http://string-db.org) is an online database used to identify interactions among DEGs. A confidence score $\geq 0.7$ was set for conducting the PPI network. Cytoscape (version3.7.1), is an open-source bioinformatics software platform, used for visualizing the PPI network and further analyses. The plugin-in Molecular Complex Detection (MCODE) of Cytoscape was performed to identify significant modules based on the topology of the PPI network. The criteria were set as follows: degree cut-off $=2$, node score cut-off $=0.2, \mathrm{~K}$-core $=2$, Max. depth $=100$. The plug-in apps, ClueGO, were used to analyze and visualize the biological processes in significant modules.

\section{Identification of Hub genes and analysis}

The plugin-in cytoHubba of Cytoscape was used to identify hub genes based on the degree and bottleneck algorithms. Oncomine database is an online platform that computes gene expression signatures, clusters, and gene-set modules. Gene Expression Profiling Interactive Analysis (GEPIA) database is a newly developed web server for cancer and normal gene expression profiling and interactive analyses ${ }^{[14]}$. The most significant hub gene was identified by cytoHubba as a key gene and its expression pattern evaluated in common human malignancies using Oncomine and GEPIA databases.

The clinicopathological features and types of KIT/PDGFRa mutations were extracted from GSE136755 and the raw data published by Lagarde et al. ${ }^{[15]}$ The correlation of the key gene expression pattern between the clinicopathological features and types of KIT/PDGFRa mutations was determined using statistical analysis, with a $\mathrm{P}<0.05$ considered as being statistically significant.

\section{Gene set enrichment analysis (GSEA)}

GSEA is a computational method that determines whether an a priori defined gene set shows statistically significant and concordant differences between two biological states ${ }^{[16,17]}$. GSEA performs biological information from a different view and further improves the understanding of relevant biological events. GSE47911 based on the GPL6480 platform (Agilent-014850 Whole Human Genome Microarray 4x44K G4112F) was also downloaded for the GEO database and included 15 gastric GISTs. The GIST samples were divided into two groups based on the key gene expression level, and GSEA was performed subsequently. Annotated gene set (c5.bp.v6.2.symbols.gmt. [Gene oncology]) was chosen as the reference gene set. Gene size $>20$, FDR $<0.05$ and normalized enrichment score (NES) $>2.00$ were set as the cut-off criteria.

\section{Immunohistochemistry and survival analysis}

A total of 49 patients admitted to the First Affiliated Hospital of Zhejiang University (Zhejiang Province, China) between 2001 and 2015, who were diagnosed with GISTs, were enrolled in the study. Paraffinembedded GIST specimens obtained from the patients were used for perform immunohistochemical $(\mathrm{IHC})$ staining and survival analysis. None of the patients matched the following points: esection with positive margin, neoadjuvant and adjuvant imatinib treatment, and a family history of GISTs. The clinical stratification of GISTs referred to the modified NIH criteria. All tissue specimens of GISTs were provided 
by the Department of Pathology, the First Affiliated Hospital of Zhejiang University. The use of human tumor specimens and clinical data was approved by the Ethical Committee, the First Affiliated Hospital of Zhejiang University. All donors provided written informed consent while donating their tissues to this study. IHC staining was conducted as previously described ${ }^{[18]}$. Briefly, tissue sections were incubated at $4^{\circ} \mathrm{C}$ overnight with antihuman AURKA rabbit polyclonal antibody diluted 1:500 (NOVUS Biologicals, USA). A total of five adjacent fields using $\times 400$ magnifications in areas with the highest density of positive staining were scored according to the summation of the percentage of staining intensity. The percentage of immunostaining was defined as $0(<5 \%), 1(<20 \%), 2(20-50 \%)$, and $3(>50 \%)$. The staining intensity was defined from 0 (no staining) to 3 (strongest staining). The maximum score was 6 which corresponded to more than $50 \%$ of the cells with the strongest staining intensity. AURKA staining score less than the mean value was considered as low expression, while scores more than the mean value were considered as high expression. The follow-up time in all the patients was calculated from the date of surgery to the date of disease recurrence or last visit.

\section{Cell culture}

GIST cell line GIST/T1 donated by Prof. Wenbin Chen (Zhejiang University, Hangzhou, China) was obtained from the Cell Bank of the Type Culture Collection of the Chinese Academy of Sciences (Shanghai, China). The cells were maintained in PRMI-1640 medium (GIBCO) supplemented with 10\% heat-inactivated fetal bovine serum (GIBCO) in a humidified atmosphere with $5 \% \mathrm{CO}_{2}$ at $37^{\circ} \mathrm{C}$.

\section{Construction and transfection of lentiviral vectors for AURKA}

The DNA fragment encoding AURKA sequence was synthesized and inserted into the lentivirus expression vector pLVX-IRES-tdtomato (TaKaRa, China). The resulting vector was identified as pLVXAURKA-IRES-tdtomato. The lentiviral plasmids were transfected into HEK 293T cells with psPAX2 and pMD2.G in a ratio of 4:3:1 using Lipofectamine 2000 (Invitrogen, USA). The virus was collected 48h after transfection. GIST/T1 cells were infected with lentivirus for $48 \mathrm{~h}$. The transfection efficiency was assessed by PCR and western blotting.

\section{RNA extraction and real-time quantitative PCR (RT-qPCR)}

Total RNA was extracted using TRIzol reagent, followed by treatment with RQ1 RNase-Free DNase (Promega, USA). Reverse transcription reaction was carried out using PrimeScriptTM RT Master Mix (Takara, China) according to the manufacturer's instructions. RT-qPCR was performed to confirm the expression of AURKA using CFX Connect Real-Time System (BIO-RAD, USA) with the SuperReal PreMix Color SYBR Green kit (Tiangen, China). Primer sets used for RT-qPCR are shown in Table 1. Gene expression levels were normalized against the expression of actin using the $2^{-\triangle \Delta C T}$ method.

\section{Western Blotting}


Protein $(30 \mu \mathrm{g})$ from each sample were separated by $10 \%$ sodium dodecyl sulfate-polyacrylamide gel electrophoresis (SDS-PAGE), and the proteins transferred to polyvinylidene difluoride (PVDF) membrane (Merck Millipore, USA). The membrane was blocked with $5 \%$ nonfat milk or $5 \%$ BSA in TBST (tris-buffered saline with $0.1 \%$ Tween 20 ) for $1 \mathrm{~h}$ at room temperature. The membrane was incubated with a primary antibody AURKA (1:1000 dilution, NOVUS Biologicals, USA) overnight at $4{ }^{\circ} \mathrm{C}$. The membrane was washed thrice with $0.1 \%$ TBST buffer for $30 \mathrm{~min}$, followed by probing with Goat anti-Rabbit IgG-HRP secondary antibody (1:2000 dilution; Santa Cruz, USA), and the membrane washed thrice with $0.1 \%$ TBST buffer for $30 \mathrm{~min}$. The HRP conjugated second antibody was detected and visualized with the enhanced chemiluminescence detection system (GE Healthcare, USA). The band intensity was quantified by densitometry using ImageJ software (version 1.49; National Institutes of Health, USA).

\section{Proliferation assay}

Cell proliferation was assessed by CCK8 assay (Tongren Chemical Society, Japan). $3 \times 10^{5}$ cells per well were seeded in 96 -well plates and incubated in $5 \% \mathrm{CO}_{2}$ at $37^{\circ} \mathrm{C}$ for $24 \mathrm{~h}$. imatinib $(3 \mu \mathrm{M})$ was added to the culture medium for $48 \mathrm{~h}$ to investigate the effect of AURKA on imatinib resistance. The medium was discarded and replaced with serum-free medium and CCK8 (10 $\mathrm{\mu l})$ and incubated for $2 \mathrm{~h}$. The Biokinetics Reader (MD corporate, USA) was used to detect the absorbance at $450 \mathrm{~nm}$.

\section{Apoptosis assay}

Cell apoptosis was assessed using the Annexin V-APC/7-AAD apoptosis kit (MultiSciences, China). $3 \times$ $10^{5}$ cells per well were seeded in 6-well plates and incubated in $5 \% \mathrm{CO}_{2}$ at $37^{\circ} \mathrm{C}$ for $24 \mathrm{~h}$. imatinib $(3 \mu \mathrm{M})$ or solvent was added to the culture medium for $48 \mathrm{~h}$ and the cells collected. After washing twice in PBS at $4^{\circ} \mathrm{C}$, the cells were re-suspended in 500 $\mu$ l binding buffer. Annexin V-APC $(5 \mu \mathrm{l})$ and 7-AAD $(10 \mu \mathrm{l})$ were added onto the suspension and incubated for $5 \mathrm{~min}$ at $4^{\circ} \mathrm{C}$. The apoptosis index was examined by flow cytometry (ACCURI C6; BD, USA).

\section{Migration assay}

Cell migration was assessed in 24-well transwell chambers with upper and bottom culture compartments separated by a polycarbonate membrane with $8 \mu \mathrm{m}$ pore size. $7.5 \times 10^{4}$ cells at logarithmic phage were seeded in the upper chamber. The culture medium with/without imatinib $(3 \mu \mathrm{M})$ was added to the bottom chamber. The cells were incubated for $72 \mathrm{~h}$ and fixed in $4 \%$ paraformaldehyde. The non-migration cells were washed and the inserts air-dried. The cells were incubated in $200 \mu 1 \%$ crystal violet for $10 \mathrm{~min}$ at room temperature. The number of cells that invaded the gel were counted in 3 fields $(\times 100)$ using an inverted microscope (IX73; OLYMPUS, Japan).

\section{Statistical analysis}

All statistical analyses were performed using the SPSS 22.0 package (SPSS Inc., USA). Descriptive data were expressed as mean \pm SD. The comparison of data among multiple groups was evaluated by two- 
way analysis of variance (ANOVA) and student's t-test. Kaplan-Meier method was performed to determine the disease-free survival (DFS) curves, and the log-rank test was used for survival curve comparison. COX proportional hazard model was used to perform multivariate analyses, and the Forward Likelihood Ratio method was used to identify independent variables. $P<0.05$ was considered to be statistically significant.

\section{Results}

\section{Identification of DEGs}

DEGs screening from GSE136755 was performed using GEO2R for the defined advanced and nonadvanced GIST samples. A total of 606 genes were identified using the cut-off criteria of adj. $P<0.05$ and Log2FoldChange $>1$, including 244 upregulated and 362 downregulated genes. The top 50 up- and downregulated genes were presented in a heatmap (Figure 1A).

\section{Functional annotation of DEGs}

To explore the biological clustering of DEGs, GO and KEGG analyses for the up- and downregulated DEGs were performed using DAVID. The upregulated DEGs based on $G O$ analysis were found to be significantly enriched in cell division, sister chromatid cohesion, mitotic nuclear division, microtubule-based movement, and mitotic metaphase plate congression. The cellular component (CC) of the genes was significantly enriched in midbody, kinesin complex, spindle, spindle microtubule, and condensed chromosome kinetochore. The molecular function (MF) of the genes was significantly enriched in microtubule motor activity and microtubule-binding. KEGG analysis showed that the genes were mainly involved in cell cycle and oocyte meiosis. The downregulated DEGs, based on GO analysis showed that they were significantly enriched in the interferon-gamma-mediated signaling pathway, type I interferon signaling pathway, antigen processing and presentation, antigen processing and presentation of peptide antigen via MHC class I and antigen processing and presentation of exogenous peptide antigen via MHC class I and TAP-independent antigen presentation on MHC class I molecules. The CC of the genes was significantly enriched in the integral component of the lumenal side of the endoplasmic reticulum membrane and ER to Golgi transport vesicle membrane. The MF of the genes was significantly enriched in peptide antigen binding and MHC class II receptor activity. KEGG analysis further showed that the genes were mainly involved in Graft-versus-host disease, Type I diabetes mellitus, Allograft rejection, Antigen processing and presentation, and Viral myocarditis. The results are shown in Figure 1B.

\section{Module analysis through PPI network of DEGs}

To identify the significant modules, STRING was performed on the PPI network of DEGs which composed of 603 nodes and 1768 edges. The PPI enrichment P-value $<1.0 \mathrm{E}-16$. The PPI network was visualized by Cytoscape and further analyzed by plug-in MCODE. Module 1 and module 2, were reserved and purely contained the upregulated and downregulated DEGs, respectively. In module 1, GO analysis showed that the genes were significantly enriched in cell cycle-related biological processes and signaling pathways. 
For module 2, GO analysis showed that the genes were significantly enriched in immunological processes and signaling pathways. The results are shown in Figure 2A.

\section{Identification of hub genes}

The top 15 hub genes filtered according to the degree algorithm were CDK1, KIF11, KIF2C, CENPE, KIF20A, BUB1, CCNA2, CCNB1, AURKA, MAD2L 1, CDCA8, KIF4A, CENPF, NDC80, and KIF23, with the scores ranging from 58 to 48 . The top 15 hub genes filtered according to the bottleneck algorithm were $A U R K A$, FN1, CD44, VEGFA, IL6, HLA-DQB1, HLA-DPA1, CXCL8, NT5E, ANK2, FOXM1, CHEK1, STAT1, CDC25A, and IFIH1, with the scores ranging from 64 to 8 . The Venn diagram was used to identify the most significant hub genes. The results showed that AURKA was the only common hub gene in the two hub gene cohorts and was thus considered as a key gene. The results are shown in Figure 2B.

\section{GSEA}

GSE47911 gene profiles were divided into groups downloaded and GSEA performed based on AURKA expression level. The samples with the highest expression (25\%, 4 samples) and lowest expression (25\%, 4 samples) were selected to further analysis using GSEA. The results indicated that cell cycle-related gene sets were associated with high expression of AURKA (Figure 3A).

\section{AURKA expression pattern in common human malignancies}

To determine whether AURKA is common in other human malignancies, the mRNA expression level of AURKA in stomach carcinoma, liver hepatocellular carcinoma, and colorectal carcinoma, was evaluated using data from TCGA. The results showed that AURKA was significantly upregulated in all the above malignancies. The results from the Oncomine database also supported the upregulated expression of AURKA in most human malignancies (Figure 3B).

\section{Correlation between AURKA expression and clinicopathological features in GISTs}

To determine the clinical significance of AURKA expression in GISTs, AURKA expression level was assessed in 49 GIST tissues using IHC staining (Figure 4). The correlation between AURKA expression with clinicopathological features (age, gender, location, risk stratification) was determined (Table 2). This study found that AURKA expression was closely associated with tumor risk stratification (Figure 4; $\mathrm{P}<$ 0.001). The clinical significance of AURKA expression in GISTs was also evaluated in GSE136755 and raw data published by Lagarde et al. ${ }^{[19]}$ (Table 3, Table 4, and Figure 5). The results from GSE136755 showed a significant association of AURKA expression with risk stratification $(\mathrm{P}<0.001)$ and stage $(\mathrm{P}<$ 0.001). Raw data published by Lagarde et al. ${ }^{[19]}$ also showed a significant association of AURKA expression with risk stratification $(P<0.001)$. However, except for data from GSE136755 which revealed a significant correlation between AURKA expression and tumor location $(\mathrm{P}=0.018)$, both our and Lagarde's data found no significant correlation between AURKA expression and tumor location. Notably, the 
significant correlation seen in GSE136755 data diminished when the tumor location was subclassified into gastric, small intestinal, large intestinal and metastatic GISTs $(P=0.607$, supplement).

To determine the prognostic significance of AURKA expression in GISTs, Kaplan-Meier survival analysis was performed. The range of observation time was 9 - 79 months. As shown in Figure 4, patients with high AURKA expression had poorer DFS than those with low AURKA expression (43.25 \pm 6.94 months vs $98.48 \pm 3.44$ months $\triangle \mathrm{P}<0.001)$. COX proportional hazard model showed that AURKA can be useful as an independent prognostic factor in GISTs $(P=0.002)$.

Gene mutation types can predict the response of GISTs to imatinib. GISTs with KIT exon 11, PDGFRa exon 12, and PDGFRa exon 14 mutations were considered to be the most sensitive to imatinib. GISTs with other mutations such as KIT exon 9, KIT exon 13, KIT exon 14, KIT exon 17, KIT exon 18, and PDGFRa exon 18 D842V and KIT/PDGFRa wild type GISTs were insensitive/resistant to imatinib ${ }^{[1,3,20]}$. In the GSE136755, containing 56 imatinib sensitive and 7 imatinib-resistant GISTs, no significant correlation between AURKA expression and imatinib-resistant gene mutations was found $(P=0.074)$. Fortunately, Lagarde et al. provided more data on imatinib resistance associated with KIT/PDGFRa mutations ( $\mathrm{n}=$ 15). There was a significant correlation between AURKA expression and imatinib-resistant gene mutations $(P=0.018)$. The results are shown in Figure 5, Table 2, and Table 3.

\section{AURKA overexpression promotes GIST/T1 cell proliferation, survival, and migration}

To assess the biological effects of AURKA in GISTs, AURKA was overexpressed in GIST/T1 cells and transfected with the AURKA-expressing virus, which was defined as the AURKA overexpression group (AURKA group). The normal GIST/T1 cells (Blank group) and the GIST/T1 cells transfected with vacant plasmids (Vectorgroup) were considered as the control groups. The transfection efficiency was determined by the red fluorescence from tdtomato, and quantified by RT-qPCR and western blotting. As shown in Figure 6, compared with the Blank and Vector groups, AURKA was overexpressed in GIST/T1 cells transfected with AURKA-expressing virus (the AURKA group).

CCK8 assay was used to assess the effects of AURKA on cell proliferation. The results showed that AURKA overexpression significantly increased the cell proliferation of GIST/T1 cells in comparison with the Blank and Vector groups $(P=0.018)$ (Figure $7 A)$. Imatinib treatment significantly inhibited cell proliferation in all three groups. However, compared with the control groups, AURKA overexpressed cells showed a relatively higher proliferation level in the presence of imatinib $(P<0.001)$ (Figure 7A). This indicated that AURKA overexpression can promote cell proliferation of GIST/T1 cells.

In this study, the regulation of cell apoptosis by AURKA overexpression in GIST/T1 cells was investigated by Annexin $V$ assay. The results from flow cytometry demonstrated that AURKA overexpression markedly suppressed the apoptotic process in GIST/T1 cells $(P<0.001)$ (Figure 7B). In the Blank group, treatment with imatinib slightly promoted cell apoptosis $(P<0.001)$. Similar results were observed in the Vector group $(\mathrm{P}=0.002)$ and $A U R K A$ group $(\mathrm{P}=0.026)$. These results confirmed the effect of imatinib on apoptosis of GIST cells. Treatment of the AURKA group with imatinib revealed that cell apoptosis rate 
was significantly lower in AURKA overexpressed GIST/T1 cells compared to the control imatinib-treated Blank and Vectorgroups $(\mathrm{P}<0.001)$ (Figure 7B). Further, these results revealed that AURKA overexpression enhanced the imatinib resistance of GIST/T1 cells.

Besides cell proliferation and cell apoptosis, the effect of AURKA overexpression on cell migration was also examined. The number of AURKA overexpressed cells that passed through the membrane was significantly increased than that of the other two control groups (for AURKA group, rate $=47.00 \pm 5.06 \%$; for Blank group, rate $=26.17 \pm 3.66 \% \bigotimes$ for Vector group, rate $=30.00 \pm 4.15 \%)$ in the absence of imatinib (for both Blank and Vectorgroup, $\mathrm{P}<0.001$ ) (Figure $7 \mathrm{C}$ ). However, imatinib significantly inhibited cell migration in all three groups $(P<0.001)$ and effectively eliminated the effect of AURKA on cell migration (Figure $7 C, P=0.169$ ). The quantitative analysis of the number of migrating cells is shown in Figure $7 C$. These findings indicated that AURKA overexpression could promote GIST/T1 cell migration, however, this was inhibited by imatinib treatment.

\section{Discussion}

GIST is a rare mesenchymal malignancy originating in the human alimentary system. GIST is known to possess specific biological features compared to other carcinomas. Lymph node metastasis is not common in GISTs and occurs preferably in young patients ${ }^{[21,22]}$. A 1- or 2-cm macroscopic margin may be sufficient to achieve microscopically negative margins ${ }^{[23]}$. GIST has a poor response to conventional chemotherapy and radiotherapy ${ }^{[24]}$. Therefore, exploring key genes that regulate the biological behavior of GIST can better understand the characteristics of GIST and formulate better therapeutic strategies. In recent years, several therapeutic targets have been explored. The emergence and development of bioinformatics have been useful in exploring molecular targets for the progression and prognosis of $\mathrm{GIST}^{[25-27]}$.

In this study, the gene profiles of GSE136755, including 65 GIST samples not treated with preoperative imatinib were analyzed. The gene signatures of GISTs with KIT mutations were analyzed. Comparison of the gene profiles of advanced GISTs with non-advanced GISTs generated 244 upregulated DEGs and 362 downregulated DEGs. Functional annotation showed that the upregulated DEGs were mainly enriched in cell cycle-related biological processes and pathways, such as cell division (GO term), sister chromatid cohesion (GO term), mitotic nuclear division (GO term), cell cycle (KEGG term) and oocyte meiosis (KEGG term). Otherwise, the downregulated DEGs were mainly enriched in immune-related biological processes and pathways, such as interferon-gamma-mediated signaling pathway (GO term), type I interferon signaling pathway (GO term), antigen processing and presentation (GO term), Graft-versus-host disease (KEGG term) and Antigen processing and presentation (KEGG term). STRING database and Cytoscape software were applied for further exploration of DEGs. Two important modules were extracted and visualized. The genes in module 1 were associated with the upregulated DEGs and mainly involved in cell cycle related biological processes and signaling pathways, while those in module 2 were associated with the downregulated DEGs and mainly involved in immunological processes and signaling pathways. The 
results indicated the different gene profiles between advanced and non-advanced GISTs were mainly involved in the cell cycle and tumor immunity.

Plug-in cytoHubba in Cytoscape software was used based on the degree and bottleneck algorithms to screen for novel key genes associated with GIST progression. The degree algorithm calculates the relevance and abundance of genes, while the bottleneck algorithm evaluates key gene positions in an entire regulatory network. In this study, a significant key gene, AURKA, was identified using a Venn diagram. AURKA is a protein-coding gene that encodes a cell cycle-regulated kinase involved in microtubule formation and/or stabilization at the spindle pole during chromosome segregation. Previous studies have shown that AURKA promotes tumor progression via enhancing cell cycle progression, apoptosis, genomic instability, epithelial-mesenchymal transition (EMT) and stem-like properties of cancer cells. ${ }^{[28]}$ In most solid tumors, AURKA regulates cell cycle checkpoints and promotes cell cycle procession. ${ }^{[28]}$ The results of GSEA based on gene profiles of GSE47911 validated the association between AURKA overexpression and the cell cycle procession.

To confirm the importance of AURKA expression in GISTs, we performed IHC staining to analyze the correlation of AURKA expression and clinicopathological characteristics of GISTs among enrolled 49 GIST patients. The results showed that there were high levels of AURKA expression in advanced GISTs. These results were consistent with the data provided by GSE136755 and Lagarde et al. ${ }^{[19]}$ Survival analysis further showed that AURKA overexpression could be an independent prognostic factor for the clinical outcome of GISTs. A series of in vitro experiments subsequently showed that GIST cells with AURKA overexpression had a significant advantage in malignant behavior, such as cell proliferation, antiapoptosis and migration. That validated the results of bioinformatics analysis.

Drug resistance is a major obstacle in cancer chemotherapy and greatly affects a patient's prognosis. Adjuvant imatinib has been widely used as a first-line treatment in patients with advanced GIST $[29,30]$. However, imatinib resistance has increased in recent years. By extracting raw data published by Lagarde et al. ${ }^{[19]}$, a significant relationship was found between AURKA overexpression and mutations on the genes causing imatinib-resistance. GSE136755 data showed a similar but not significant result about AURKA overexpression and imatinib-resistance related gene mutations, which could be likely due to the small data size. Besides, in vitro experiments showed that AURKA overexpression reduced the sensitivity of GIST cells to imatinib by promoting cell proliferation and inhibiting apoptosis. This suggested that AURKA decreased the response of GIST cells to imatinib, hence there was an association between AURKA overexpression and imatinib resistance.

The role of AURKA overexpression in tumor progression has been reported in other human malignancies. AURKA phosphorylates RPS6KB1 and promotes cell proliferation and survival of gastrointestinal cancer cells $^{[31]}$. AURKA also stabilizes the transcription factor N-MYC, thereby promoting G1/S cell cycle transition and tumor cell proliferation ${ }^{[32]}$. Pharmacological inhibition of AURKA promoted the chemosensitivity of cervical cancer cells ${ }^{[33]}$. Compounds targeting AURKA, particularly alisertib, have been extensively studied in preclinical models, where they demonstrate synergy with other targeted 
therapies, leading to tumor regression in a variety of cancer models ${ }^{[34]}$. Lagarde et al. ${ }^{[19]}$ reported that CINSARC (Complexity Index in SARComa) score and AURKA expression are prognostic factors for GIST and that AURKA may be an interesting potential therapeutic target ${ }^{[15]}$. Yeh et al. confirmed the contribution of AURKA inhibitor, MLN8237, in suppressing metastatic GISTs ${ }^{[35]}$. This study revealed the important role of AURKA in tumor progression and imatinib resistance GISTs.

This study had the following limitations. First, being a retrospective study, there was selection bias in the enrolled GIST patients. Second, the gene mutation data in the enrolled GISTs were not available. Lagarde et al. reported a significant correlation between AURKA overexpression and imatinib-resistant gene mutations. Third, this study did not explore AURKA related signaling pathways in GIST. However, bioinformatics analyses indicated that AURKA promoted cell cycle-related signaling pathways. Future research should focus on exploring the detailed mechanisms between AURKA and GIST progression.

\section{Conclusions}

In conclusion, this study identifies AURKA as a key gene in GIST progression and illustrates the contribution of AURKA in imatinib-resistant GIST cells. These findings further suggest that AURKA is a promising treatment target for GISTs. Further in vitro and in vivo experiments are needed to explore and validate the detailed AURKA-mediated signaling pathways in GIST progression.

\section{Declarations}

\section{Ethic approval and consent to participate}

This study was approved by the Ethical Committee of the First Affiliated Hospital, Zhejiang University School of Medicine.

\section{Consent for publication}

Not applicable.

\section{Availability of data and materials}

All data and materials generated and analyzed during this study are included in the main paper.

\section{Competing interests}

The authors declare no conflict of interest.

\section{Funding}

This research was supported by Zhejiang Provincial Natural Science Foundation of China under Grant No. LY15H160045. 
Author's contributes

The work presented here was carried out through collaborative efforts of many authors. Conception and design: XC and WW. Bioinformatic analyses: XC and JW. In vitro experiments: WF and SL. Acquisition, analysis and interpretation of data: XC. Writing, review, and revision of the manuscript: XC and WW. All authors read and approved the final manuscript.

\section{Acknowledgement}

We acknowledge and appreciate Prof. Wenbin Chen for donating GIST/T1 cell line.

\section{References}

1. Corless CL, Barnett CM, Heinrich MC. Gastrointestinal stromal tumours: origin and molecular oncology. Nat Rev Cancer. 2011;11:865-78.

2. Wozniak A, Gebreyohannes YK, Debiec-Rychter M, Schoffski P. New targets and therapies for gastrointestinal stromal tumors. Expert Rev Anticancer Ther. 2017;17:1117-29.

3. Liu P, Tan F, Liu H, Li B, Lei T, Zhao X. The Use of Molecular Subtypes for Precision Therapy of Recurrent and Metastatic Gastrointestinal Stromal Tumor. Onco Targets Ther. 2020;13:2433-47.

4. Joensuu $\mathrm{H}$. Risk stratification of patients diagnosed with gastrointestinal stromal tumor. Hum Pathol. 2008;39:1411-9.

5. Iorio N, Sawaya RA, Friedenberg FK. Review article: the biology, diagnosis and management of gastrointestinal stromal tumours. Aliment Pharmacol Ther. 2014;39:1376-86.

6. Valsangkar N, Sehdev A, Misra S, Zimmers TA, O'Neil BH, Koniaris LG. Current management of gastrointestinal stromal tumors: Surgery, current biomarkers, mutations, and therapy. Surgery. 2015;158:1149-64.

7. Serrano C, George S, Valverde C, Olivares D, Garcia-Valverde A, Suarez C, et al. Novel Insights into the Treatment of Imatinib-Resistant Gastrointestinal Stromal Tumors. Target Oncol. 2017;12:277-88.

8. Bauer S, Yu LK, Demetri GD, Fletcher JA. Heat shock protein 90 inhibition in imatinib-resistant gastrointestinal stromal tumor. Cancer Res. 2006;66:9153-61.

9. Belinsky MG, Rink L, Cai KQ, Ochs MF, Eisenberg B, Huang M, et al. The insulin-like growth factor system as a potential therapeutic target in gastrointestinal stromal tumors. Cell Cycle. 2008;7:294955.

10. Demetri GD, van Oosterom AT, Garrett CR, Blackstein ME, Shah MH, Verweij J, et al. Efficacy and safety of sunitinib in patients with advanced gastrointestinal stromal tumour after failure of imatinib: a randomised controlled trial. Lancet. 2006;368:1329-38.

11. Demetri GD, Reichardt P, Kang YK, Blay JY, Rutkowski P, Gelderblom H, et al. Efficacy and safety of regorafenib for advanced gastrointestinal stromal tumours after failure of imatinib and sunitinib 
(GRID): an international, multicentre, randomised, placebo-controlled, phase 3 trial. Lancet. 2013;381:295-302.

12. Zhao X, Xu M, Cai Z, Yuan W, Cui W, Li MD. Identification of LIFR, PIK3R1, and MMP12 as Novel Prognostic Signatures in Gallbladder Cancer Using Network-Based Module Analysis. Frontiers in Oncology 2019;9.

13. Yang JF, Shi SN, Xu WH, Qiu YH, Zheng JZ, Yu K, et al. Screening, identification and validation of CCND1 and PECAM1/CD31 for predicting prognosis in renal cell carcinoma patients. Aging. 2019;11:12057-79.

14. Tang Z, Li C, Kang B, Gao G, Li C, Zhang Z. GEPIA: a web server for cancer and normal gene expression profiling and interactive analyses. Nucleic Acids Res. 2017;45:W98-102.

15. Lagarde P, Perot G, Kauffmann A, Brulard C, Dapremont V, Hostein I, et al. Mitotic Checkpoints and Chromosome Instability Are Strong Predictors of Clinical Outcome in Gastrointestinal Stromal Tumors. Clin Cancer Res. 2011;18:826-38.

16. Subramanian A, Tamayo P, Mootha VK, Mukherjee S, Ebert BL, Gillette MA, et al. Gene set enrichment analysis: a knowledge-based approach for interpreting genome-wide expression profiles. Proc Natl Acad Sci U S A. 2005;102:15545-50.

17. Mootha VK, Lindgren CM, Eriksson KF, Subramanian A, Sihag S, Lehar J, et al. PGC-1alpharesponsive genes involved in oxidative phosphorylation are coordinately downregulated in human diabetes. Nat Genet. 2003;34:267-73.

18. Chen WB, Cheng XB, Ding W, Wang YJ, Chen D, Wang JH, et al. Centromere protein F and survivin are associated with high risk and a poor prognosis in colorectal gastrointestinal stromal tumours. $\mathrm{J}$ Clin Pathol. 2011;64:751-5.

19. Lagarde P, Perot G, Kauffmann A, Brulard C, Dapremont V, Hostein I, et al. Mitotic checkpoints and chromosome instability are strong predictors of clinical outcome in gastrointestinal stromal tumors. Clin Cancer Res. 2012;18:826-38.

20. Corless CL, Schroeder A, Griffith D, Town A, McGreevey L, Harrell P, et al. PDGFRA mutations in gastrointestinal stromal tumors: frequency, spectrum and in vitro sensitivity to imatinib. J Clin Oncol. 2005;23:5357-64.

21. von Mehren M, Joensuu H. Gastrointestinal Stromal Tumors. J Clin Oncol. 2018;36:136-43.

22. Agaimy A, Wunsch PH. Lymph node metastasis in gastrointestinal stromal tumours (GIST) occurs preferentially in young patients < or $=40$ years: an overview based on our case material and the literature. Langenbecks Arch Surg. 2009;394:375-81.

23. Joensuu H, Hohenberger P, Corless CL. Gastrointestinal stromal tumour. Lancet. 2013;382:973-83.

24. Keung EZ, Fairweather M, Raut CP. The Role of Surgery in Metastatic Gastrointestinal Stromal Tumors. Curr Treat Options Oncol. 2016;17:8.

25. Ohshima K, Fujiya K, Nagashima T, Ohnami S, Hatakeyama K, Urakami K, et al. Driver gene alterations and activated signaling pathways toward malignant progression of gastrointestinal stromal tumors. Cancer Sci. 2019;110:3821-33. 
26. Chen JS, Yeh CN, Cheng CT, Yen CC, Chen YY, Huang SC, et al. Role of PLK1 signaling pathway genes in gastrointestinal stromal tumors. Oncol Lett. 2018;16:3070-82.

27. Amirnasr A, Gits CMM, van Kuijk PF, Smid M, Vriends ALM, Rutkowski P, et al. Molecular Comparison of Imatinib-Naive and Resistant Gastrointestinal Stromal Tumors: Differentially Expressed microRNAs and mRNAs. Cancers (Basel) 2019;11.

28. Yan $M$, Wang $C$, He B, Yang M, Tong M, Long Z, et al. Aurora-A Kinase: A Potent Oncogene and Target for Cancer Therapy. Med Res Rev. 2016;36:1036-79.

29. Dematteo RP, Ballman KV, Antonescu CR, Maki RG, Pisters PW, Demetri GD, et al. Adjuvant imatinib mesylate after resection of localised, primary gastrointestinal stromal tumour: a randomised, doubleblind, placebo-controlled trial. Lancet. 2009;373:1097-104.

30. Joensuu H, Eriksson M, Sundby Hall K, Reichardt A, Hartmann JT, Pink D, et al. Adjuvant Imatinib for High-Risk GI Stromal Tumor: Analysis of a Randomized Trial. J Clin Oncol. 2016;34:244-50.

31. Wang-Bishop L, Chen Z, Gomaa A, Lockhart AC, Salaria S, Wang J, et al. Inhibition of AURKA Reduces Proliferation and Survival of Gastrointestinal Cancer Cells With Activated KRAS by Preventing Activation of RPS6KB1. Gastroenterology. 2019;156:662-75.e7.

32. Otto T, Horn S, Brockmann M, Eilers U, Schuttrumpf L, Popov N, et al. Stabilization of N-Myc is a critical function of Aurora A in human neuroblastoma. Cancer Cell. 2009;15:67-78.

33. Sun JM, Yang LN, Xu H, Chang B, Wang HY, Yang G. Inhibition of Aurora A promotes chemosensitivity via inducing cell cycle arrest and apoptosis in cervical cancer cells. Am J Cancer Res. 2015;5:1133-45.

34. Otto T, Sicinski P. Cell cycle proteins as promising targets in cancer therapy. Nat Rev Cancer. 2017;17:93-115.

35. Yeh CN, Yen CC, Chen YY, Cheng CT, Huang SC, Chang TW, et al. Identification of aurora kinase A as an unfavorable prognostic factor and potential treatment target for metastatic gastrointestinal stromal tumors. Oncotarget. 2014;5:4071-86.

\section{Tables}

Table 1 Sequences of primers used for Quantitative real-time PCR analysis.

\begin{tabular}{|lll|}
\hline Gene & Forward (5囚-3邓) & Reverse (5囚-3囚) \\
\hline Homo AURKA & TGGGTGGTCAGTACATGCTC & TGCATCCGACCTTCAATCATTTC \\
\hline Homo Actin & AGCGAGCATCCCCCAAAGTT & GGGCACGAAGGCTCATCATT \\
\hline
\end{tabular}

Table 2 Correlation between AURKA expression level and clinical features in 49 GIST patients 


\begin{tabular}{|llllll|}
\hline Parameter & Characteristic & No. patients (\%) & AURKA score & statistics & P value \\
\hline Gender & Female & $23(46.9)$ & $1.93 \pm 1.35$ & $\mathrm{t}=-0.430$ & 0.669 \\
\hline Male & $26(53.1)$ & $2.12 \pm 1.52$ & & \\
\hline & & & & & \\
\hline Location & Stomach & $14(28.6)$ & $1.74 \pm 1.30$ & $\mathrm{~F}=1.321$ & 0.277 \\
\hline & Small intestine & $12(24.5)$ & $1.68 \pm 1.30$ & & \\
\hline & Large intestine & $23(46.9)$ & $2.37 \pm 1.53$ & & \\
\hline & & $25(51.0)$ & $2.00 \pm 1.36$ & & \\
\hline Risk* & High & $18(36.7)$ & $2.02 \pm 1.43$ & $\mathrm{~F}=35.449$ & $<0.001$ \\
\hline & Intermediate & $4(8.2)$ & $2.1 \pm 1.29$ & & \\
\hline & Low & $20(40.8)$ & $1.26 \pm 0.74$ & & \\
\hline & Very low & $7(14.3)$ & $0.40 \pm 0.52$ & & \\
\hline
\end{tabular}

*P $<0.05$ was considered statistically significant

Table 3 Correlation between AURKA intensity and clinical features based on GSE136755 


\begin{tabular}{|c|c|c|c|c|c|}
\hline Parameter & Characteristic & No. patients (\%) & AURKA intensity & statistics & $P$ value \\
\hline \multirow[t]{2}{*}{ Gender } & Female & $26(40.0)$ & $6.19 \pm 0.97$ & $t=-1.458$ & 0.150 \\
\hline & Male & $39(60.0)$ & $6.63 \pm 1.47$ & & \\
\hline \multirow[t]{2}{*}{ Age } & $<68$ & $31(47.7)$ & $6.28 \pm 1.17$ & $t=1.022$ & 0.311 \\
\hline & $\geq 68$ & $34(52.3)$ & $6.62 \pm 1.41$ & & \\
\hline \multirow[t]{3}{*}{ Location* } & Stomach & $43(66.2)$ & $6.14 \pm 1.25$ & $F=4.306$ & 0.018 \\
\hline & Non-stomach & $16(24.6)$ & $6.95 \pm 0.99$ & & \\
\hline & Metastasis & $6(9.2)$ & $7.39 \pm 1.74$ & & \\
\hline \multirow[t]{4}{*}{ Risk* } & High & $17(26.2)$ & $7.54 \pm 1.30$ & $F=11.898$ & $<0.001$ \\
\hline & Intermediate & $9(13.8)$ & $5.75 \pm 0.54$ & & \\
\hline & Low & $22(33.8)$ & $6.06 \pm 0.94$ & & \\
\hline & Very low & $11(16.9)$ & $5.66 \pm 0.74$ & & \\
\hline \multirow[t]{4}{*}{ Stage* } & IV & $9(13.8)$ & $7.94 \pm 1.63$ & $F=10.686$ & $<0.001$ \\
\hline & III & $9(13.8)$ & $6.85 \pm 0.89$ & & \\
\hline & $\|$ & $8(12.3)$ & $7.13 \pm 1.55$ & & \\
\hline & I & $39(60.0)$ & $5.89 \pm 0.84$ & & \\
\hline \multirow[t]{3}{*}{ Mutation } & sensitive & $56(86.2)$ & $6.39 \pm 1.21$ & $F=2.541$ & 0.087 \\
\hline & resistance & $7(10.8)$ & $7.33 \pm 1.84$ & & \\
\hline & $\mathrm{N} / \mathrm{A}$ & $2(3.1)$ & $5.29 \pm 0.20$ & & \\
\hline \multirow[t]{2}{*}{ Mutation } & Sensitive & $56(88.9)$ & $6.39 \pm 1.21$ & $t=1.814$ & 0.075 \\
\hline & Resistance & $7(11.1)$ & $7.33 \pm 1.84$ & & \\
\hline
\end{tabular}

$* \mathrm{P}<0.05$ was considered statistically significant 
Table 4 Correlation between AURKA intensity and clinical features based on the published raw data

\begin{tabular}{|c|c|c|c|c|c|}
\hline Parameter & Characteristic & No. patients (\%) & AURKA intensity & statistics & $P$ value \\
\hline \multirow[t]{3}{*}{ Location } & Stomach & $40(66.7)$ & $8.91 \pm 1.25$ & $F=1.923$ & 0.156 \\
\hline & Non-stomach & $16(26.7)$ & $9.64 \pm 1.55$ & & \\
\hline & Parenteral & $4(6.7)$ & $9.47 \pm 1.69$ & & \\
\hline \multirow[t]{4}{*}{ AFIP Risk* } & High & $17(28.3)$ & $10.2 \pm 1.59$ & $F=8.686$ & $<0.001$ \\
\hline & Intermediate & $14(23.3)$ & $9.38 \pm 1.43$ & & \\
\hline & Low & $16(26.7)$ & $8.48 \pm 0.64$ & & \\
\hline & Very low & $13(21.7)$ & $8.34 \pm 0.55$ & & \\
\hline \multirow[t]{2}{*}{ Local recurrence* } & No & $54(90.0)$ & $8.83 \pm 1.03$ & $t=7.377$ & $<0.001$ \\
\hline & Yes & $6(10.0)$ & $12.0 \pm 0.63$ & & \\
\hline \multirow[t]{2}{*}{ Metastasis* } & No & $45(75.0)$ & $8.56 \pm 0.82$ & $t=6.921$ & $<0.001$ \\
\hline & Yes & $15(25.0)$ & $10.9 \pm 1.22$ & & \\
\hline \multirow[t]{2}{*}{ Mutation* } & Sensitive & $45(75.0)$ & $8.91 \pm 0.18$ & $t=2.442$ & 0.018 \\
\hline & Resistance & $15(25.0)$ & $9.88 \pm 0.44$ & & \\
\hline
\end{tabular}

${ }^{*} \mathrm{P}<0.05$ was considered statistically significant

\section{Figures}


A

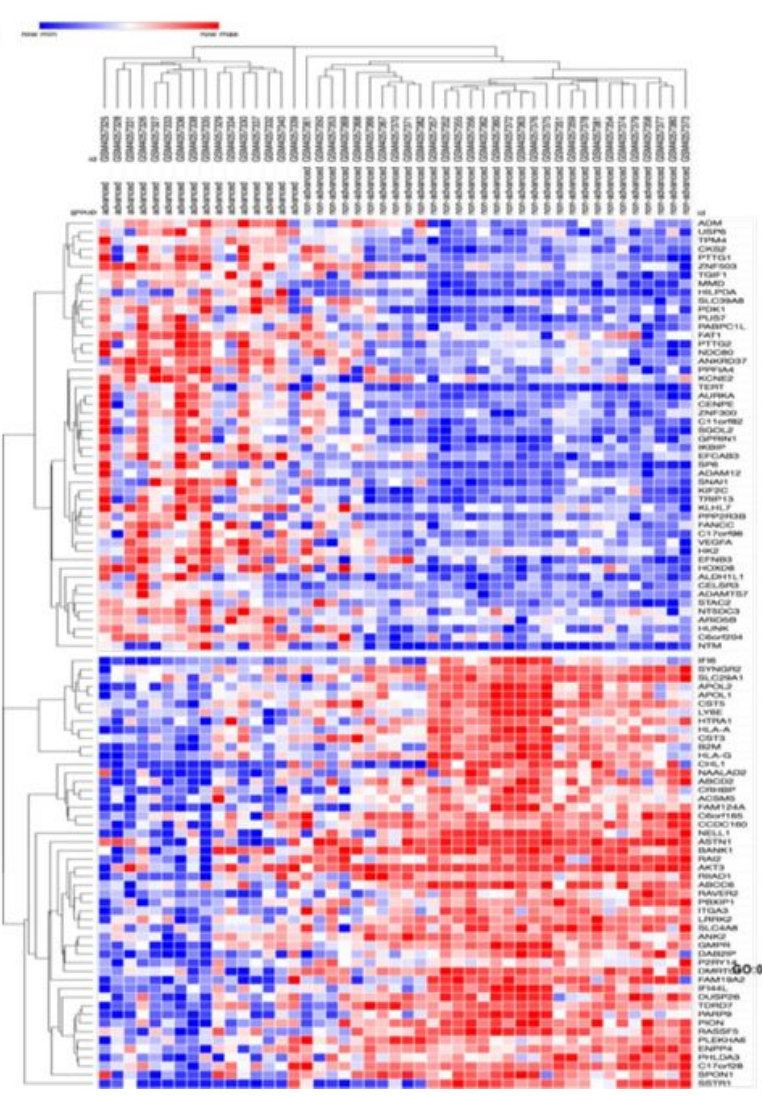

B
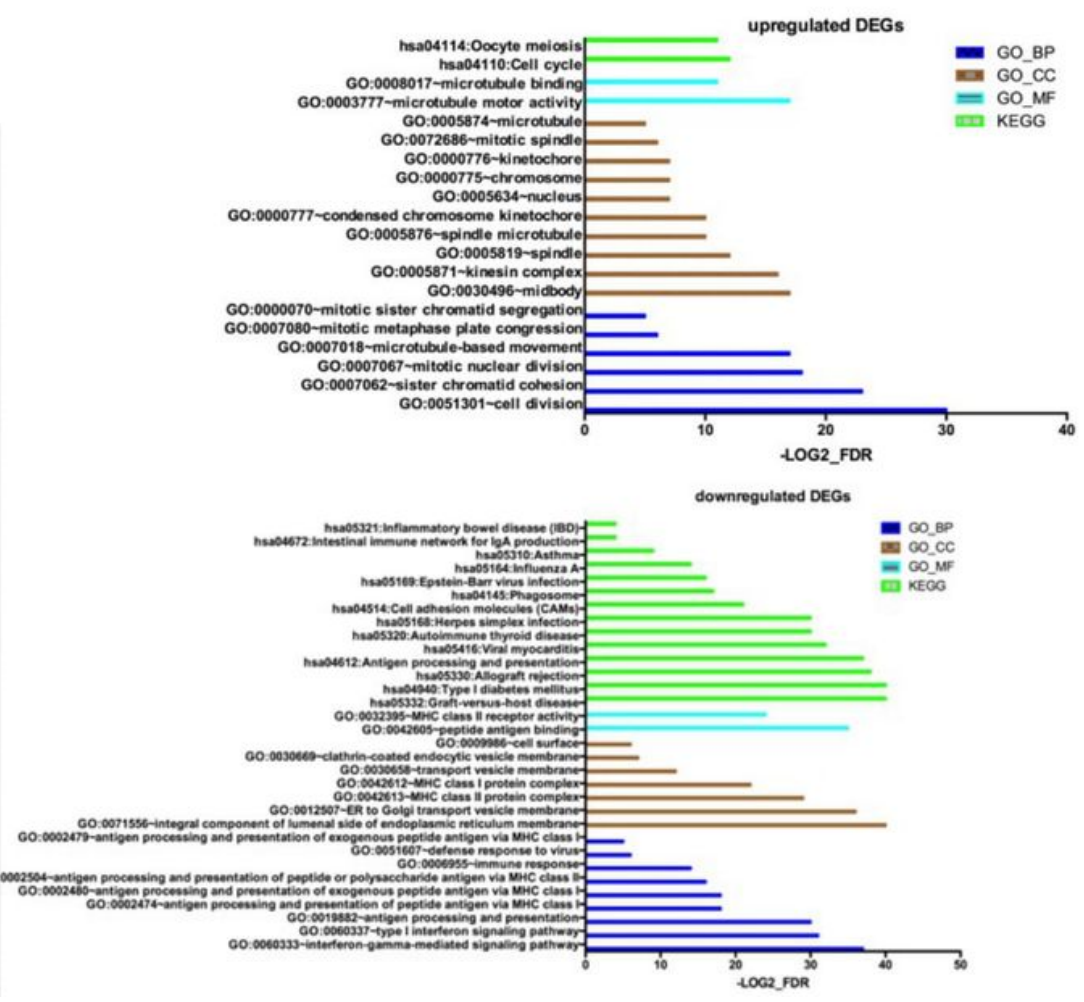

\section{Figure 1}

Screening of DEGs from GSE136755. (A) Heat map generated based on the top 50 up-and downregulated DEGs between advanced and non-advanced GIST samples. (B) Functional annotation of DEGs by $\mathrm{GO}$ and KEGG enrichment analyses.
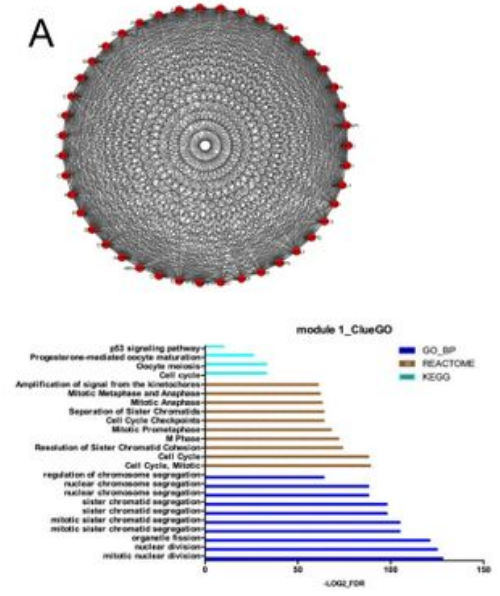
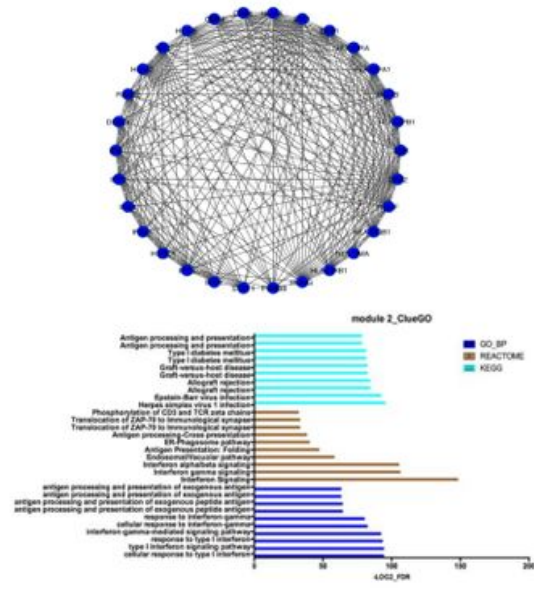
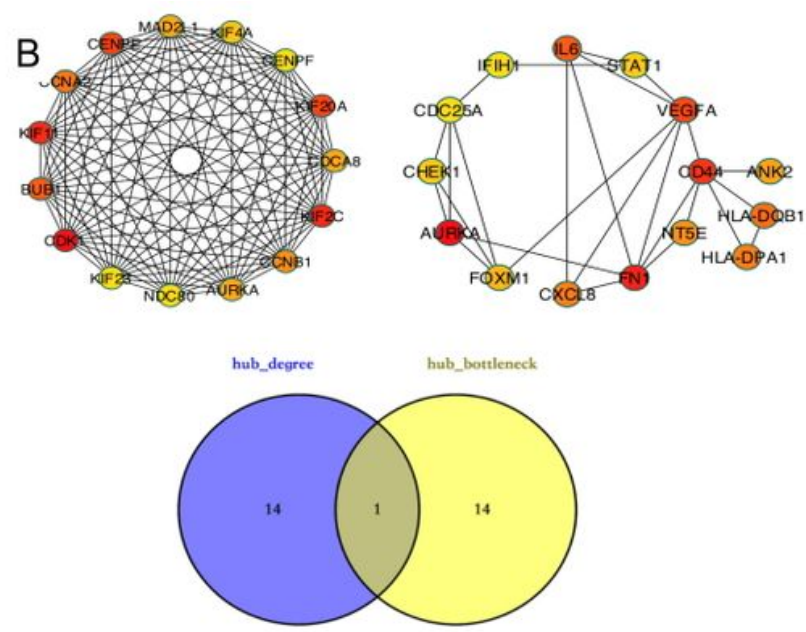

Figure 2

Module analysis and identification of hub genes from GSE136755. (A) The most significant modules based on the topology in the PPI network identified using Molecular Complex Detection (MCODE) of Cytoscape. Module 1 contains upregulated DEGs (red color), while module 2 contains downregulated 
DEGs (blue color). The functional annotation of modules 1 and 2 determined by ClueGO analyses. (B) Hub genes identified using CytoHubba of Cytoscape based on the degree and bottleneck algorithms. The most significant hub gene in the Venn diagram is considered as a key gene.

A
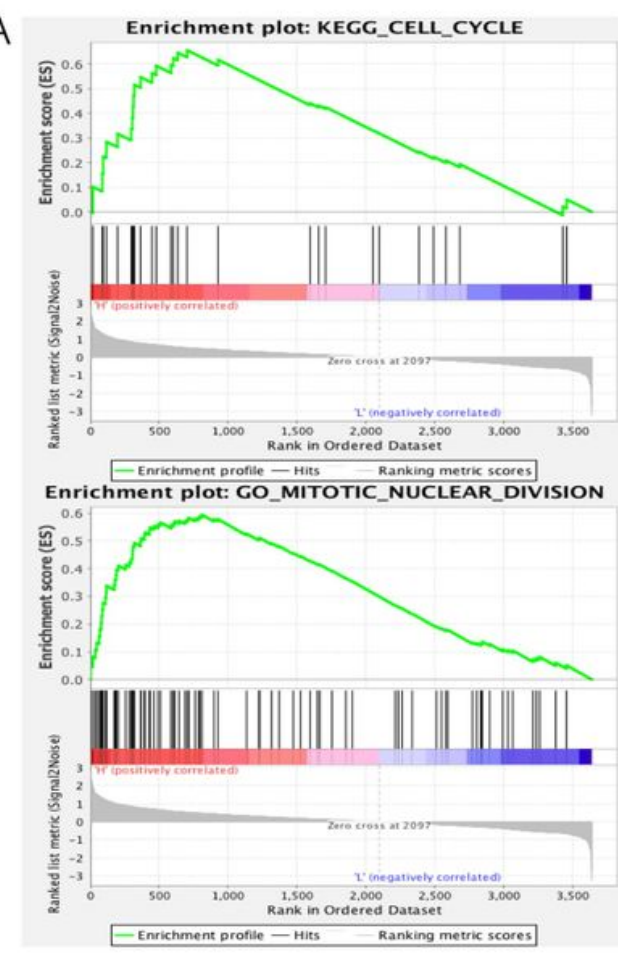

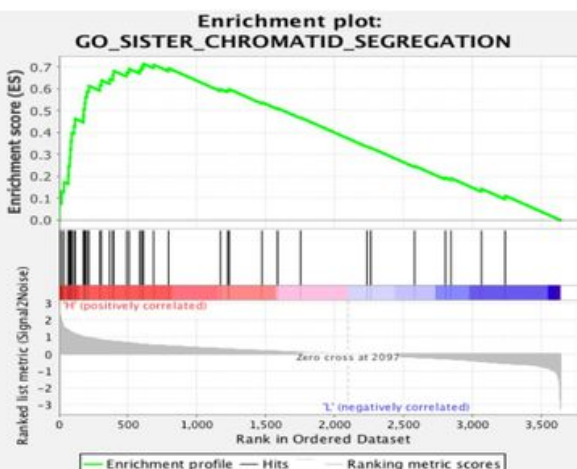

Enrichment profile - Hits Ranking metric scores
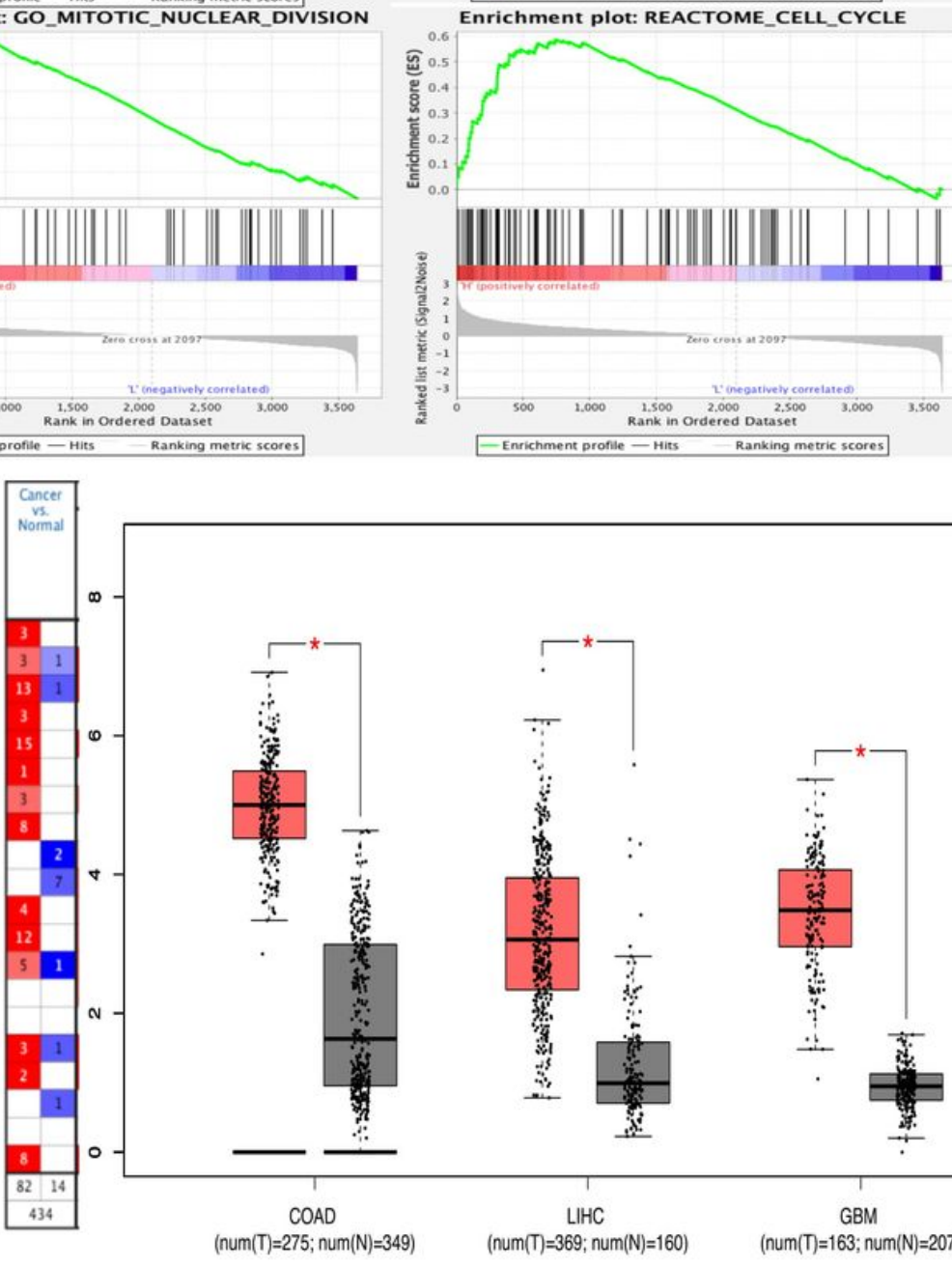

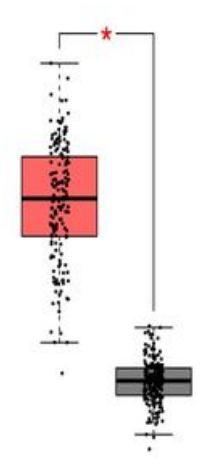

$\stackrel{\text { LHHC }}{\operatorname{Lin}(T)=369 ; \operatorname{num}(\mathrm{N})=160)}$
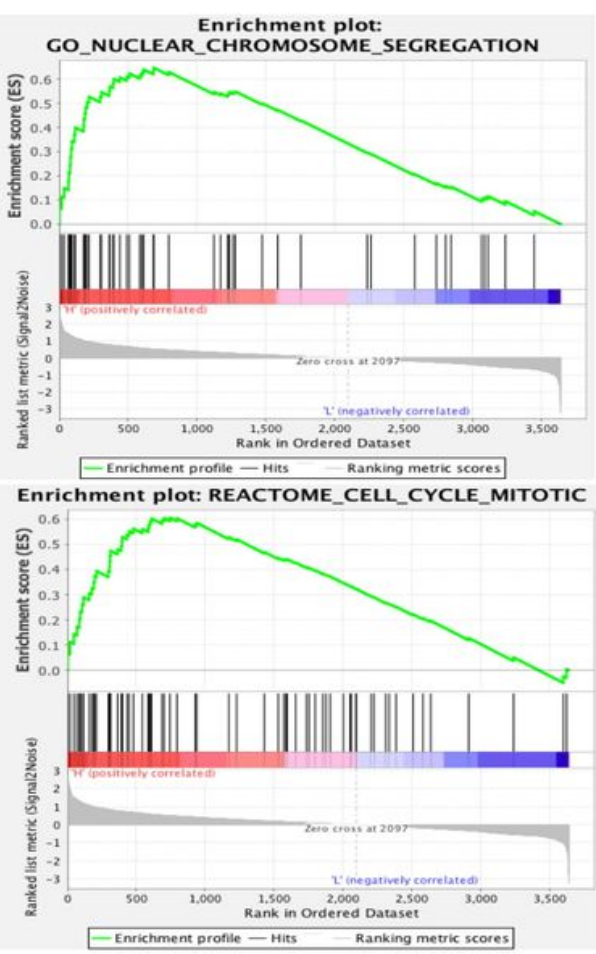

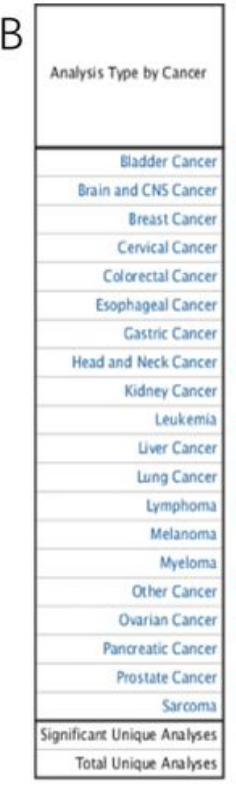

$(\operatorname{num}(\mathrm{T})=275 ; \operatorname{num}(\mathrm{N})=349)$

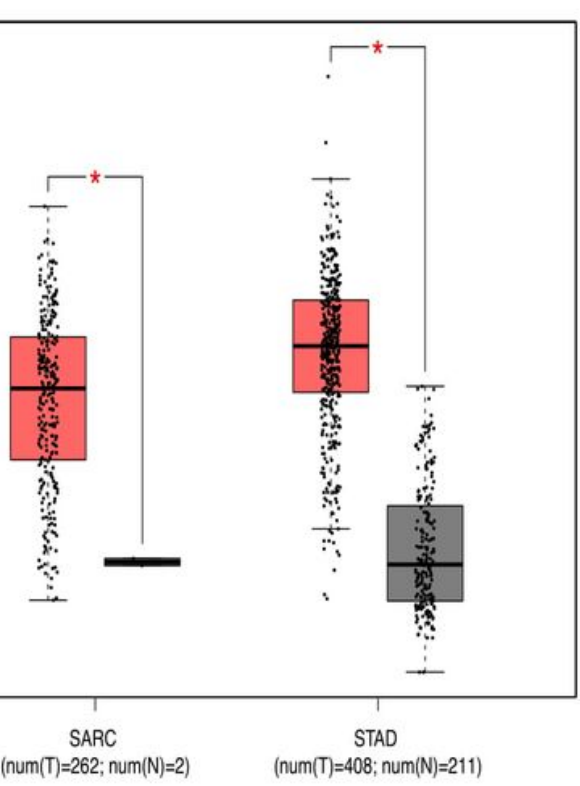

\section{Figure 3}

Validation of AURKA expression in GSE47911 and different human malignancies. (A) Representative gene set enrichment analysis (GSEA) for GSE47911 showing that gene sets related to the cell cycle are associated with high expression of AURKA. (B) Expression patterns of upregulated AURKA in several human malignancies based on Oncomine and GEPIA databases. 
A

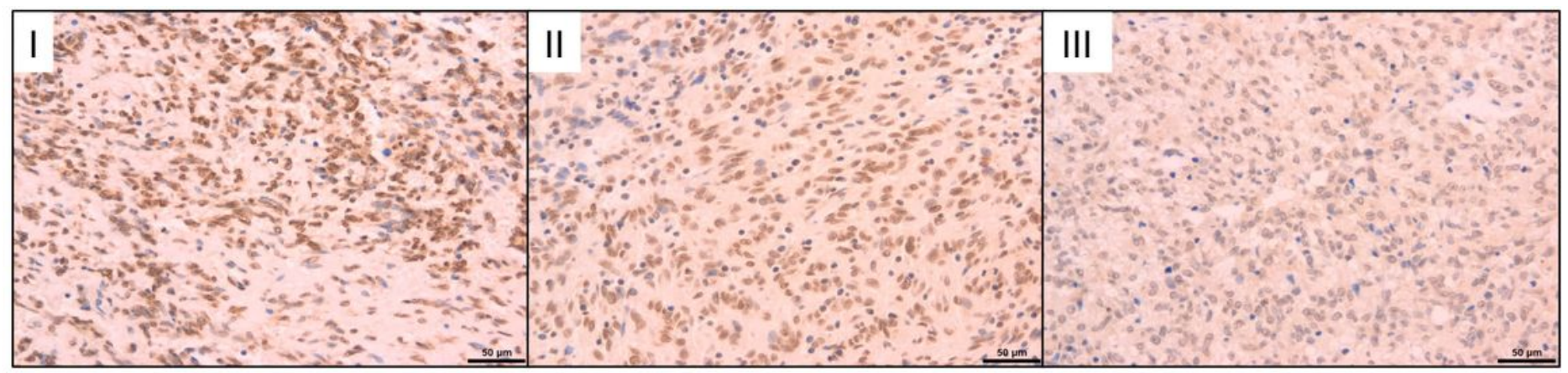

B

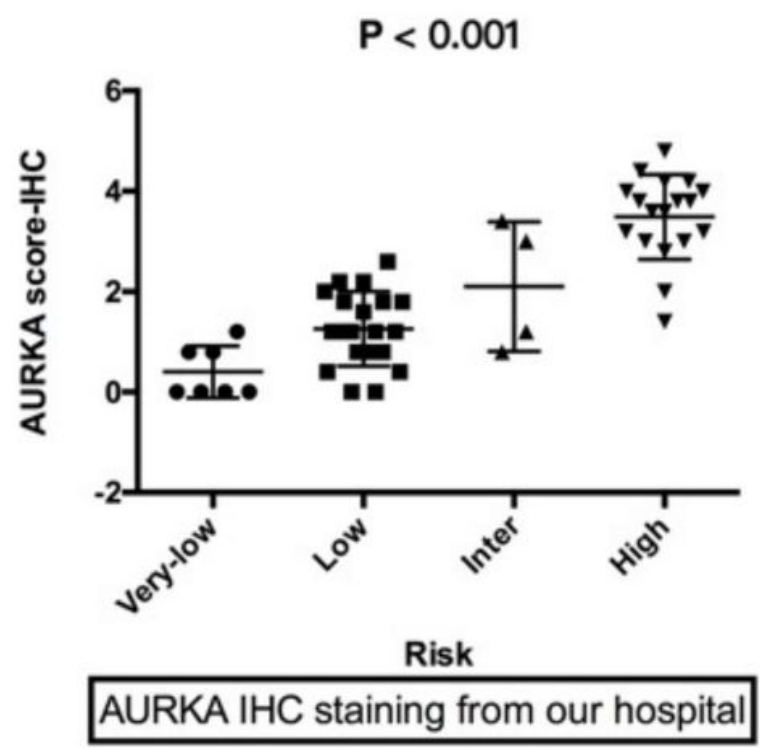

C

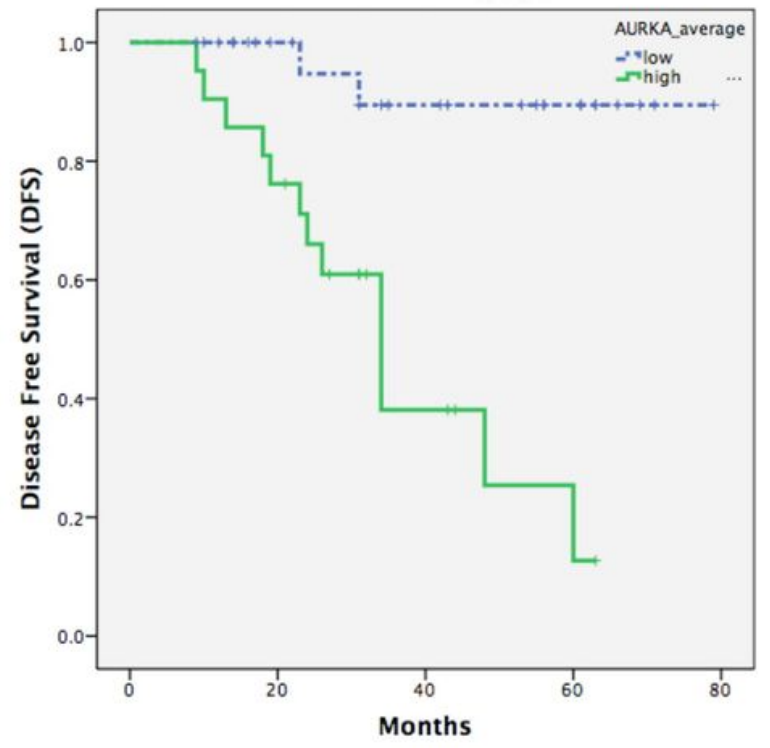

Figure 4

Expression of AURKA in GIST patients. (A) Immunohistochemical (IHC) staining of GIST specimens. I: strong staining, II: intermediate staining, III: weak staining. (B) IHC scores of AURKA varied across different risk groups of GISTs, with high-risk GISTs showing the highest expression of AURKA. (C) Patients with high AURKA expression exhibited poorer DFS than those with low AURKA expression.

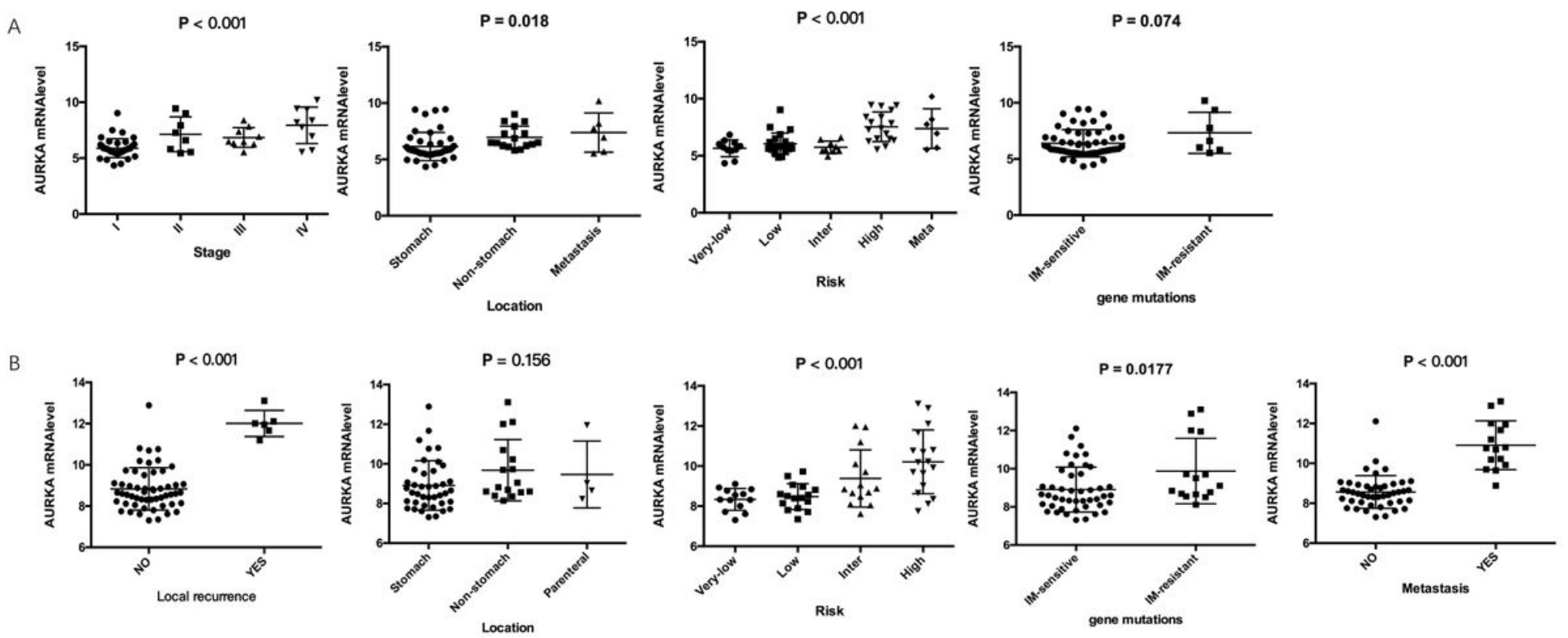




\section{Figure 5}

Expression profiles of AURKA in different GIST cohorts. (A) AURKA expression is significantly associated with tumor location, stage and risk stratification based on data from GSE136755, while there is no obvious correlation of AURKA expression to imatinib-related gene mutations (B) The raw data published by Lagarde et al. (ref.) shows that AURKA expression is significantly associated with risk stratification and imatinib-related gene mutations. There is also an obvious correlation of AURKA expression to clinical outcome, such as local recurrence and metastasis. AURKA expression shows no significant difference among gastric, non-gastric and parenteral GISTs.

A
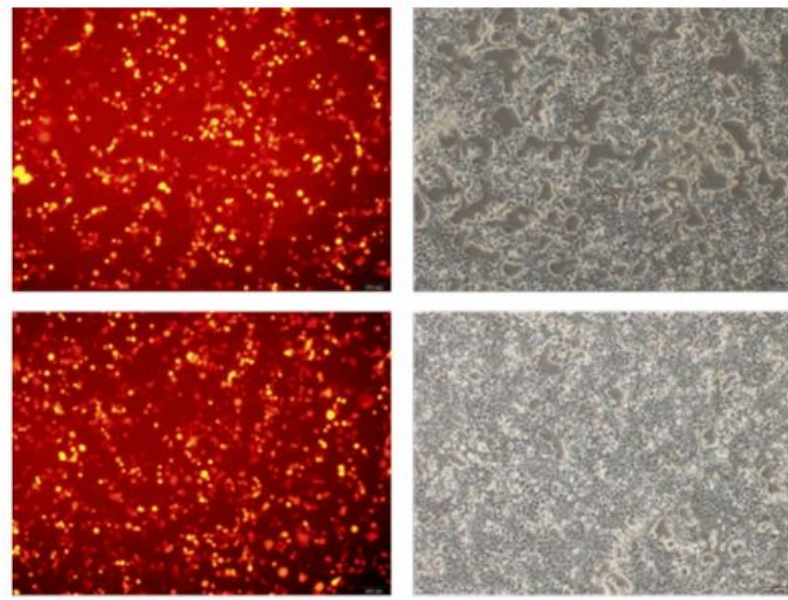

AURKA

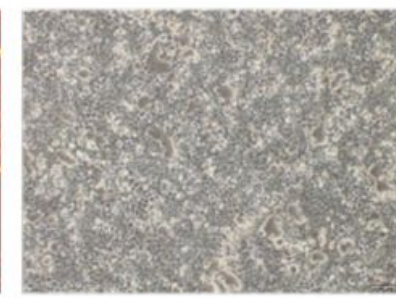

Vector

$P<0.001$

B

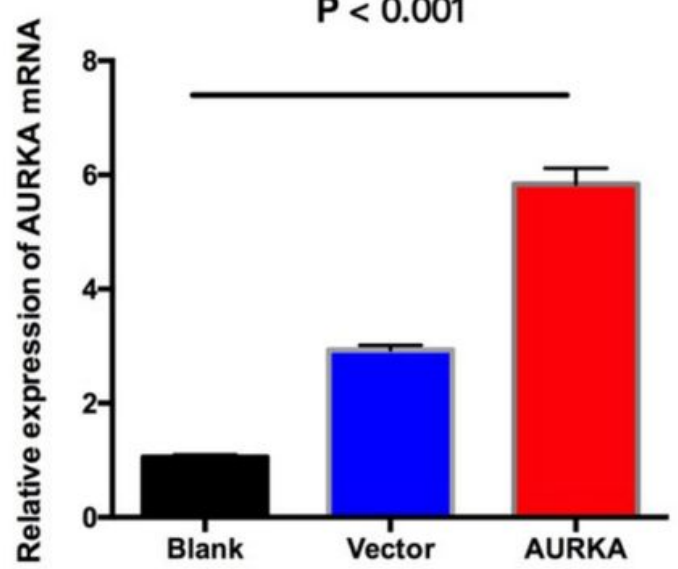

C
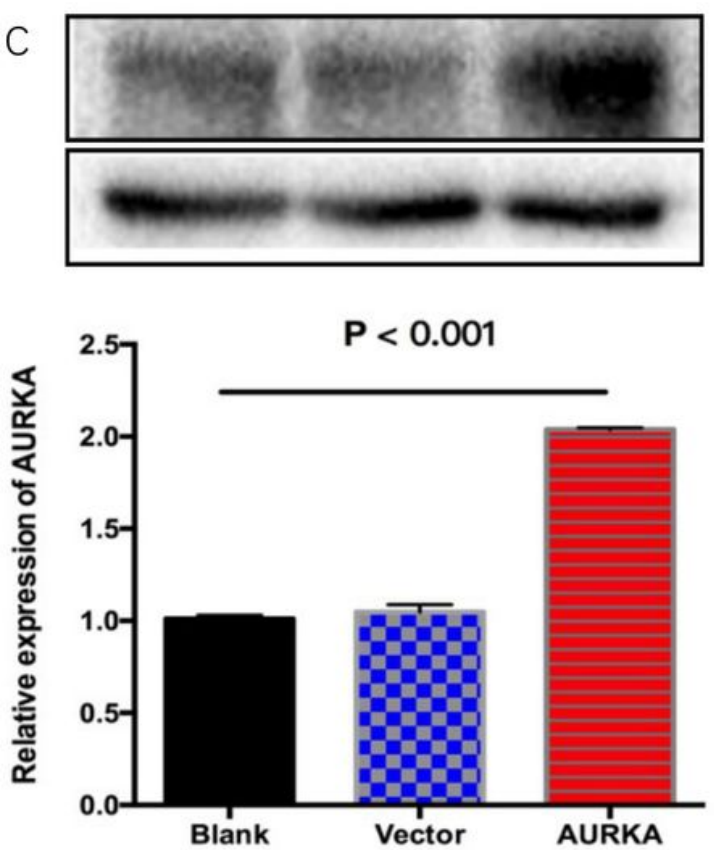

\section{Figure 6}

Construction of GIST/T1 cells with overexpressed AURKA. (A) Representative images of cells transfected with AURKA plasmids and control plasmids captured with fluorescent and bright field microscopy. (B) mRNA level of AURKA are significantly higher in AURKA group compared to the Blank and Vector groups. (C) Protein levels of AURKA are higher in AURKA group than in the Blank and Vector groups. 

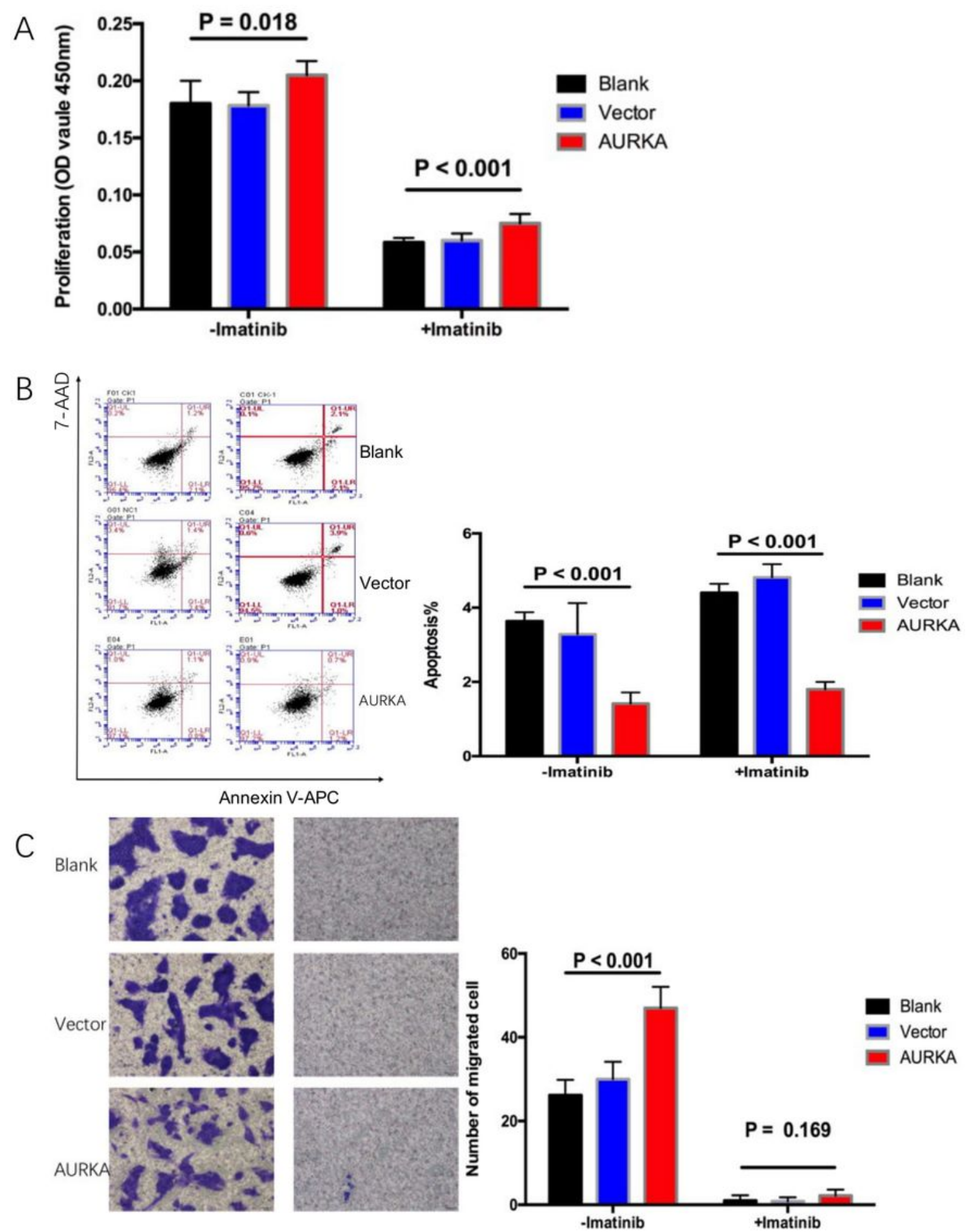

\section{Figure 7}

The effect of AURKA overexpression on aggressive behavior of GIST cells as revealed by in vitro experiments. (A) AURKA overexpression promotes cell proliferation of GIST/T1 cells with/without imatinib intervention. (B) Annexin $V$ assay reveals that AURKA overexpression improves cell survival and enhances the imatinib resistance in GIST/T1 cells. (C) Representative images of crystal violet staining showing the number of migrating cells in the Blank, Vector, and AURKA groups with/without imatinib 
intervention. AURKA overexpression promotes GIST/T1 cell migration and this effect is eliminated by imatinib treatment. 